\title{
Blind Data Detection in the Presence of PLL Phase Noise by Sequential Monte Carlo Method
}

\author{
Erdal Panayırci ${ }^{\dagger}$, Hakan A. Çırpan ${ }^{\ddagger}$, Marc Moeneclaey ${ }^{\S}$ and Nele Noels ${ }^{\S}$ \\ ${ }^{\dagger}$ Department of Electronics Engineering, Bilkent University, Bilkent 06800, Ankara, Turkey \\ ${ }^{\ddagger}$ Department of Electrical Engineering, Istanbul University, Avcilar 34850, Istanbul, Turkey \\ $\S$ TELIN/DIGCOM Department, Ghent University, B9000 Gent, Belgium
}

\begin{abstract}
In this paper, based on a sequential Monte Carlo method, a computationally efficient algorithm is presented for blind data detection in the presence of residual phase noise generated at the output the phase tracking loop employed in a digital receiver. The basic idea is to treat the transmitted symbols as " missing data" and draw samples sequentially of them based on the observed signal samples up to time $t$. This way, the Bayesian estimates of the phase noise and the incoming data are obtained through these samples, sequentially drawn,together with their importance weights. The proposed receiver structure is seen to be ideally suited for high-speed parallel implementation using VLSI technology.
\end{abstract}

\section{INTRODUCTION}

Carrier phase synchronization is a critical issue in coherent digital communication systems. A considerable amount of research has been carried out for data detection in the presence of the time-varying phase noise as well as the fixed phase offset [1]. Estimating the phase offset and detecting the data jointly by maximum likelihood (ML) technique does not seem to be analytically tractable. Even if the likelihood function can be evaluated offline, however, it is invariably a nonlinear function of the parameter to be estimated, which makes the maximization step (which must be performed in real-time) computationally infeasible. A number of suboptimal algorithms have thus been proposed, most of which employ a two-stage receiver structure with a phase noise estimation stage followed by the data detection [2]. Phase synchronization is typically implemented by a decision directed(or data aided) or non-decision directed (or non-data aided). Decision directed schemes depend on availability of reliably detected symbol for obtaining the phase estimate, and therefore, they usually require transmission of pilot or training data. However, in applications where bandwidth is the most precious resource, training data can significantly reduce the overall system capacity. Thus blind or non-data aided techniques become an attractive alternative [3], [4].

In order to provide an implementable solution to blind techniques, recently there have been a substantial amount of work on iterative formulation of the parameter estimation problem based on the Expectation-Maximization (EM) technique [5].

This research has been conducted within the NEWCOM Network of Excellence in Wireless Communications funded through the EC 6th Framework Programme.
It is known that the EM algorithm derives iteratively and converges to the true ML estimation of these unknown parameters. The main drawbacks of this approach are that the algorithm is sensitive to the initial starting values chosen for the parameters, it does not necessarily converge to the global extremum and the convergence can be slow. Furthermore, in situation where the posterior distribution must be constantly updated with arrival of the new data with missing parts, EM algorithm can be highly inefficient, because the whole iteration process must be redone with additional data. The sequential Monte Carlo (SMC) methodology [6], [12] that has emerged in the field of statistics and engineering has shown great promise to solve such problems. This technique can approximate the optimal solution directly without compromising the system model. Additionally, the decision made at time $t$ does not depend on any decisions made previously, and thus, no error is propagated in their implementation. More importantly, the SMC yields a fully blind algorithm and allows for both Gaussian and non-Gaussian ambient noise as well as high-speed parallel implementations. Furthermore, the tracking the time-varying phase noise and the data detection are naturally integrated. The algorithm is selfadaptive and no training/pilot symbols or decision feedback are needed [11], [7].

The main objective of this paper is to investigate the use of the SMC method to the problem of jointly detecting the data and estimating the residual phase noise, generated at the output of the a phase-locked-loop (PLL) employed in the digital receivers. The paper is an extension of the work [7] which models the phase noise as a Wiener process, a crude approximation of the phase noise obtained at the output of a PLL. The algorithm is based on a Bayesian formulation. The basic idea is to treat the transmitted symbols as "missing data" and to sequentially draw samples of them based on the current observation and computing appropriate importance sampling weights. The technique does not require iterations and updating with new data can be done cheaply.

\section{SYSTEM DESCRIPTION}

We consider a channel-coded communication system in the presence of random phase noise and the additive Gaussian noise. The input binary information bit $d_{t}$ are encoded using 
some channel code, resulting in a code bit stream $b_{t}$. The code bits are passed to a symbol mapper, yielding complex data symbols $s_{t}$, which take values from a finite alphabet set $A=\left\{a_{1}, a_{2}, \cdots, a_{|A|}\right\}$, where $|A|$ represents the cardinality of the set $A$. Each data symbol is then transmitted through a channel whose input-output relationship is given by

$$
y_{t}=s_{t} e^{j \theta_{t}}+n_{t}, \quad t=0,1, \cdots
$$

where $y_{t}, s_{t}, \theta_{t}$, are the received signal, the transmitted symbols and the phase noise, respectively, and $n_{t}$ the additive complex Gaussian noise with mean zero and the variance $\sigma_{n}^{2}=E\left[\left|n_{t}\right|^{2}\right]$. The residual phase noise process $\theta_{t}$ at $t$ th sampling instant generated at the output of a digital phase-locked-loop can be modelled by the output of of an ARMA system driven by white Gaussian noise.

An ARMA system is defined as

$$
H(z)=\frac{\Theta(z)}{U(z)}=\frac{\sum_{n=0}^{q} \beta_{k} z^{-n}}{1+\sum_{m=1}^{p} \alpha_{k} z^{-m}}
$$

whose input and output at time $t$ are denoted by $u_{t}$ and $\theta_{t}$, respectively, where $q \leq p-1$. Defining $D$ as the shift operator $D^{k} u_{t}=u_{t-k}$, we have the following relationship

$$
\left\{\theta_{t}\right\}=H(D)\left\{u_{t}\right\}
$$

where $\left\{u_{t}\right\}$ is a sequence of independent and identically distributed (i.i.d.) zero-mean random variables with variance equal to $\sigma_{u}^{2}$. It is assumed that $u_{t}$ and $n_{t}$ are independent. The coefficients $\left\{\beta_{k}\right\}$ and $\left\{\alpha_{k}\right\}$ as well as the orders $q$ and $p$ are chosen so that the transfer function of the system matches the closed-loop transfer function of a digital PLL. A digital PLL is described by its closed-loop transfer function, $H_{C L}(z)$ as

$$
H_{C L}(z)=\frac{F(z)}{z-1+F(z)}
$$

where $F(z)$ represents the loop filter transfer function. The transfer function of the ARMA system representing the digital PLL can be determined by [1]

$$
H(z)=\frac{1-H_{C L}(z)}{z-1} .
$$

For example, for a first-order loop, $F(z)=a$ (scalar $0<a<$ 1) and from (4) it follows that

$$
H(z)=\frac{z^{-1}}{1-(1-a) z^{-1}}
$$

We now express the general observation and phase noise generation equations (1) and (3) in a state-space model as follows: Define $\left\{x_{t}\right\}=\Theta^{-1}(D)\left\{\theta_{t}\right\}$. It then follows that

$$
U(D)\left\{x_{t}\right\}=\left\{u_{t}\right\}, \quad \Theta(D)\left\{x_{t}\right\}=\left\{\theta_{t}\right\}
$$

Writing (5) explicitly we have

$$
x_{t}=-\sum_{k=1}^{p} \alpha_{k} x_{t-k}+u_{t}, \quad \theta_{t}=\sum_{k=0}^{q} \beta_{k} x_{t-k}
$$

Equations in (6) allows us to write the Kalman state equations as follows: Denoting $\boldsymbol{x}_{t}=\left[x_{t}, x_{t-1}, \cdots x_{t-p+1}\right]^{T}$, (6) can be expressed as $\boldsymbol{x}_{t}=\boldsymbol{A} \boldsymbol{x}_{t-1}+\boldsymbol{b} u_{t}$ and $\theta_{t}=\boldsymbol{c}^{T} \boldsymbol{x}_{t}$ where

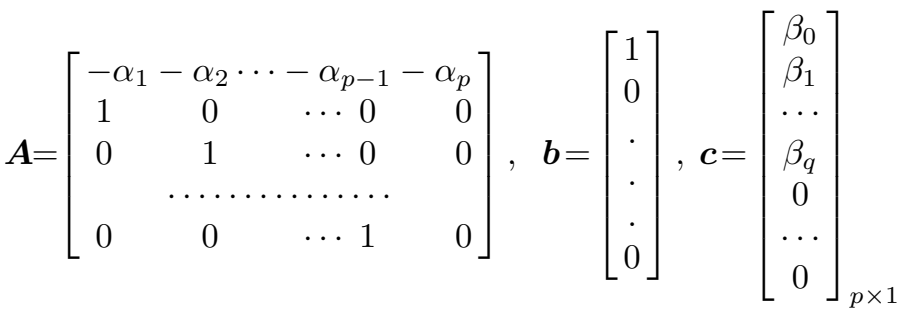

Finally, we have the following non-linear state-space model for the system

$$
\begin{aligned}
\boldsymbol{x}_{t} & =\boldsymbol{A} \boldsymbol{x}_{t-1}+\boldsymbol{b} u_{t} \\
y_{t} & =s_{t} e^{j \boldsymbol{c}^{T} \boldsymbol{x}_{t}}+n_{t}, \quad t=0,1, \cdots
\end{aligned}
$$

Our main objective is to solve the problem of online detection of the symbols $s_{t}$ and estimation of the phase noise $\theta_{t}$, completely blindly, based on the received signals up to time $t,\left\{y_{i}\right\}_{i=0}^{t}$. Defining the vectors, $\boldsymbol{S}_{t}=\left[s_{0}, s_{1}, \cdots, s_{t}\right]^{T}$, $\boldsymbol{Y}_{\mathbf{t}}=\left[y_{0}, y_{1}, \cdots y_{t}\right]^{T}, \boldsymbol{\theta}_{t}=\left[\theta_{0}, \theta_{1}, \cdots, \theta_{t}\right]^{T}$, the problem may be formulated by making Bayesian inference with respect to the posterior distribution $p\left(\boldsymbol{\theta}_{t}, \boldsymbol{S}_{t} \mid \boldsymbol{Y}_{t}\right)$.

Although this joint distribution can be written out explicitly up to a normalizing constant, the computation of the corresponding marginal joint distributions $p\left(s_{t}, \boldsymbol{x}_{t} \mid \boldsymbol{Y}_{t}\right)$, necessary for online joint symbol detection and phase noise estimation involve very high dimensional integration. Therefore, the task is mathematically infeasible in practice. The Gibbs samples [8] is a Monte Carlo method for overcoming this difficulty. However it is not an adaptive procedure and has difficulty dealing with sequentially observed data. With new data coming the whole computation must be repeated to incorporate new information. In the following section, we present an adaptive blind algorithm for the joint symbol detection and the phase noise estimation which is based on a Bayesian formulation of the problem called SMC method first developed by [8].

\section{SMC TECHNIQUE FOR BLIND DETECTION AND ESTIMATION}

We first consider the case of uncoded system, where the symbols are assumed to independent and identically distributed. i.e.

$$
P\left(s_{t}=a_{i} \mid \boldsymbol{S}_{t-1}\right)=P\left(s_{t}=a_{i}\right), a_{i} \in A
$$

For simplicity the symbols are chosen from a QPSK constellation.When no prior information about the symbols is available, 
the symbols are assumed to take each possible value in $A$ with equal probability. i.e., $P\left(s_{t}=a_{i}\right)=1 /|A|$. Since we are interested in jointly estimating the symbol $s_{t}$ and the phase noise $\boldsymbol{x}_{t}$, at time $t$ based on the observation $\boldsymbol{Y}_{t}$, the Bayes solution requires the posterior distribution

$$
p\left(s_{t}, \boldsymbol{x}_{t} \mid \boldsymbol{Y}_{t}\right)=\int p\left(\boldsymbol{x}_{t} \mid \boldsymbol{Y}_{t}, \boldsymbol{S}_{t}\right) p\left(\boldsymbol{S}_{t} \mid \boldsymbol{Y}_{t}\right) d \boldsymbol{S}_{t-1} .
$$

Note that with a given $\boldsymbol{S}_{t}$, the nonlinear (Kalman filter) model (7) can be converted into a linear model by linearizing the observation equation (1) as follows [9]:

$$
\begin{aligned}
\boldsymbol{x}_{t} & =\boldsymbol{A} \boldsymbol{x}_{t-1}+\boldsymbol{b} u_{t} \\
y_{t} & =s_{t} P_{t} \boldsymbol{c}^{T} \boldsymbol{x}_{t}+s_{t} Q_{t}+n_{t}
\end{aligned}
$$

where

$$
P_{t}=j e^{j \boldsymbol{c}^{T} \widehat{\boldsymbol{x}}_{t \mid t-1}}, \quad Q_{t}=\left(1-j \boldsymbol{c}^{T} \widehat{\boldsymbol{x}}_{t \mid t-1}\right) e^{j \boldsymbol{c}^{T} \widehat{\boldsymbol{x}}_{t \mid t-1}} .
$$

$\widehat{\boldsymbol{x}}_{t \mid t-1}$ denotes the estimator of $\boldsymbol{x}_{t}$ based on the observations $\boldsymbol{Y}_{t-1}=\left(y_{0}, y_{1} \cdots, y_{t-1}\right)$. Then the state-space model (7) becomes a linear Gaussian system. Hence,

$$
p\left(\boldsymbol{x}_{t} \mid \boldsymbol{S}_{t}, \boldsymbol{Y}_{t}\right) \sim N\left(\boldsymbol{\mu}_{t}\left(\boldsymbol{S}_{t}\right), \boldsymbol{\Sigma}_{t}\left(\boldsymbol{S}_{t}\right)\right)
$$

where the mean vector $\boldsymbol{\mu}_{t}\left(\boldsymbol{S}_{t}\right)$ and the covariance matrix $\boldsymbol{\Sigma}_{t}\left(\boldsymbol{S}_{t}\right)$ can be obtained as follows. Denoting

$$
\boldsymbol{\mu}_{t}\left(\boldsymbol{S}_{t}\right) \triangleq \widehat{\boldsymbol{x}}_{t \mid t}, \quad \boldsymbol{\Sigma}_{t}\left(\boldsymbol{S}_{t}\right) \triangleq \boldsymbol{M}_{t \mid t}
$$

$\widehat{\boldsymbol{x}}_{t \mid t}$ and $\boldsymbol{M}_{t \mid t}$ can be calculated recursively by using the Extended Kalman Technique [[9], page 449-452] with the given $\boldsymbol{S}_{t}$ as:

$$
\begin{aligned}
\widehat{\boldsymbol{x}}_{t \mid t} & =\widehat{\boldsymbol{x}}_{t \mid t-1}+\boldsymbol{K}_{t}\left(y_{t}-s_{t} e^{j \boldsymbol{c}^{T} \widehat{\boldsymbol{x}}_{t \mid t-1}}\right) \\
\boldsymbol{M}_{t \mid t} & =\left(\boldsymbol{I}-s_{t}^{*} P_{t}^{*} \boldsymbol{K}_{t} \boldsymbol{c}_{t}^{T}\right) \boldsymbol{M}_{t \mid t-1}
\end{aligned}
$$

where

$$
\begin{aligned}
\boldsymbol{K}_{t} & =\frac{s_{t} P_{t} \boldsymbol{M}_{t \mid t-1} \boldsymbol{c}_{t}}{\boldsymbol{c}^{T} \boldsymbol{M}_{t \mid t-1} \boldsymbol{c}+\sigma_{n}^{2}}, \\
\widehat{\boldsymbol{x}}_{t \mid t-1} & =\boldsymbol{A} \widehat{\boldsymbol{x}}_{t-1 \mid t-1}, \\
\boldsymbol{M}_{t \mid t-1} & =\boldsymbol{A} \boldsymbol{M}_{t-1 \mid t-1} \boldsymbol{A}^{T}+\sigma_{u}^{2} \boldsymbol{b} \boldsymbol{b}^{T} .
\end{aligned}
$$

We can now make timely estimates of $\theta_{t}$ and detection of $s_{t}$ based on the currently available observation $\boldsymbol{Y}_{t}$, up to time $t$, blindly, as follows. With the Bayes theorem, we realize that the optimal solution to this problem is

$$
\widehat{\theta}_{t}=E\left\{\theta_{t} \mid \boldsymbol{Y}_{t}\right\}=\boldsymbol{c}^{T} \widehat{\boldsymbol{x}}_{t}
$$

where

$$
\begin{aligned}
\widehat{\boldsymbol{x}}_{t} & =E\left\{\boldsymbol{x}_{t} \mid \boldsymbol{Y}_{t}\right\}=\int \boldsymbol{x}_{t} p\left(\boldsymbol{x}_{t} \mid \boldsymbol{Y}_{t}\right) d \boldsymbol{x}_{t} \\
& =\int_{\boldsymbol{S}_{t}} \underbrace{\left[\int_{\boldsymbol{x}_{t}} \boldsymbol{x}_{t} p\left(\boldsymbol{x}_{t} \mid \boldsymbol{S}_{t}, \boldsymbol{Y}_{t}\right) d \boldsymbol{x}_{t}\right]}_{\mu_{t}\left(\boldsymbol{S}_{t}\right)} p\left(\boldsymbol{S}_{t} \mid \boldsymbol{Y}_{t}\right), d \boldsymbol{S}_{t} .
\end{aligned}
$$

It then follows that

$$
\widehat{\boldsymbol{x}}_{t}=E\left\{\boldsymbol{x}_{t} \mid \boldsymbol{Y}_{t}\right\}=\int_{\boldsymbol{S}_{t}} \mu_{t}\left(\boldsymbol{S}_{t}\right) p\left(\boldsymbol{S}_{t} \mid \boldsymbol{Y}_{t}\right) d \boldsymbol{S}_{t}
$$

Similarly, the data can be detected by the hard decisions on the symbol $s_{t}$ by

$$
\widehat{s_{t}}=\arg \max _{a_{i} \in A} P\left(s_{t}=a_{i} \mid \boldsymbol{Y}_{t}\right)
$$

where

$$
P\left(s_{t}=a_{i} \mid \boldsymbol{Y}_{t}\right)=E\left\{1\left(s_{t}=a_{i}\right) \mid \boldsymbol{Y}_{t} \cdot\right\}
$$

$1\{$.$\} in (18) is an indicator function defined as$

$$
1\left(s_{t}=a_{i}\right) \begin{cases}1 & \text { if } s_{t}=a_{i} \\ 0 & \text { otherwise }\end{cases}
$$

In most cases, an exact evaluations of the expectations (16) and (18) are analytically intractable. SMC technique can provide us an alternative way for the required computation. Specifically, following the notation adopted in [10], if we can draw $m$ independent random samples $\left\{\boldsymbol{S}_{t}^{(j)}\right\}_{j=1}^{m}$ from the distribution $p\left(\boldsymbol{S}_{t} \mid \boldsymbol{Y}_{t}\right)$, then we can approximate the quantities of interest $E\left\{\boldsymbol{x}_{t} \mid \boldsymbol{Y}_{t}\right\}$ and $E\left\{1\left(s_{t}=a_{i}\right) \mid \boldsymbol{Y}_{t}\right\}$ in (16) and (18), respectively, by

$$
\begin{gathered}
E\left\{\boldsymbol{x}_{t} \mid \boldsymbol{Y}_{t}\right\} \cong \frac{1}{m} \sum_{j=1}^{m} \mu_{t}\left(\boldsymbol{S}_{t}^{(j)}\right) \\
E\left\{1\left(s_{t}=a_{i}\right) \mid \boldsymbol{Y}_{t}\right\} \cong \frac{1}{m} \sum_{j=1}^{m} 1\left(s_{t}^{(j)}=a_{i}\right)
\end{gathered}
$$

But, usually drawing samples from $p\left(\boldsymbol{S}_{t} \mid \boldsymbol{Y}_{t}\right)$ directly is usually difficult. Instead, sample generation from some trial distribution may be easier. In this case, the idea of importance sampling can be used. Suppose a set of random samples $\left\{\boldsymbol{S}_{t}^{(j)}\right\}_{j=1}^{m}$ is generated from a trial distribution $q\left(\boldsymbol{S}_{t} \mid \boldsymbol{Y}_{t}\right)$, which

- is strictly positive, $q(. \mid)>$.0 , and

- has the domain as $p(. \mid$.$) .$

By associating the weight

$$
w_{t}^{(j)}=\frac{p\left(\boldsymbol{S}_{t}^{(j)} \mid \boldsymbol{Y}_{t}\right)}{q\left(\boldsymbol{S}_{t}^{(j)} \mid \boldsymbol{Y}_{t}\right)}
$$

to the samples, the quantities of interest, $E\left\{1\left(s_{t}=a_{i}\right) \mid \boldsymbol{Y}_{t}\right\}$ and $E\left\{\boldsymbol{x}_{t} \mid \boldsymbol{S}_{t}\right\}$ can be approximated as follows:

$$
\begin{gathered}
E\left\{\boldsymbol{x}_{t} \mid y_{t}\right\} \cong \frac{1}{W_{t}} \sum_{j=1}^{m} \boldsymbol{\mu}_{t}\left(\boldsymbol{S}_{t}^{(j)}\right) w^{(j)} \\
E\left\{1\left(s_{t}=a_{i}\right) \mid \boldsymbol{Y}_{t}\right\} \cong \frac{1}{W_{t}} \sum_{j=1}^{m} 1\left(s_{t}^{(j)}=a_{i}\right) w_{t}^{(j)}, \quad i=1,2, \cdots,|A|
\end{gathered}
$$

with $W_{t}=\sum w_{t}^{(j)}$. The pair $\left(\boldsymbol{S}_{t}^{(j)}, w_{t}^{(j)}\right), j=1,2, \cdots, m$ is called a properly weighted sample with respect to distribution 
$p\left(\boldsymbol{S}_{t} \mid \boldsymbol{Y}_{t}\right)$. Note that the samples $\boldsymbol{S}_{t}^{(j)}$ can be drawn from the distribution $q\left(\boldsymbol{S}_{t} \mid \boldsymbol{Y}_{t}\right)$ sequentially as follows. We can choose $q($.$) to satisfy$

$$
q\left(\boldsymbol{S}_{t-1} \mid \boldsymbol{Y}_{t}\right)=q\left(\boldsymbol{S}_{t-1} \mid \boldsymbol{Y}_{t-1}\right) .
$$

Then, it can be easily shown that

$$
q\left(\boldsymbol{S}_{t} \mid \boldsymbol{Y}_{t}\right)=q\left(s_{t} \mid \boldsymbol{Y}_{t}, \boldsymbol{S}_{t-1}\right) q\left(\boldsymbol{S}_{t-1} \mid \boldsymbol{Y}_{t-1}\right),
$$

and one can draw samples $s_{t}^{(j)}$ from a trial distribution $q\left(s_{t} \mid \boldsymbol{Y}_{t}, \boldsymbol{S}_{t-1}^{(j)}\right)$ and let $\boldsymbol{S}_{t}^{(j)}=\left(s_{t}^{(j)}, \boldsymbol{S}_{t-1}^{(j)}\right)$ for $t=0,1, \cdots$.

Specifically, it was shown in [11] that a suitable choice for the trial distribution is of the form:

$$
q\left(s_{t} \mid \boldsymbol{Y}_{t}, \boldsymbol{S}_{t-1}^{(j)}\right)=p\left(s_{t} \mid \boldsymbol{Y}_{t}, \boldsymbol{S}_{t-1}^{(j)}\right)
$$

For this trial distribution, it is shown in [10] that the importance weight is updated according to

$$
w_{t}^{(j)}=w_{t-1}^{(j)} p\left(y_{t} \mid \boldsymbol{Y}_{t-1}, \boldsymbol{S}_{t-1}^{(j)}\right), \quad t=0,1, \cdots
$$

The predictive distribution in (24) is given by

$$
\begin{aligned}
p\left(y_{t} \mid \boldsymbol{Y}_{t-1}, \boldsymbol{S}_{t-1}^{(j)}\right) & =\sum_{a_{i} \in A} p\left(y_{t} \mid \boldsymbol{Y}_{t-1}, \boldsymbol{S}_{t-1}^{(j)}, s_{t}=a_{i}\right) \\
& \times P\left(s_{t}=a_{i} \mid \boldsymbol{Y}_{t-1}, \boldsymbol{S}_{t-1}^{(j)}\right)
\end{aligned}
$$

where (25) holds because $s_{t}$ is independent of $\boldsymbol{S}_{t-1}$ and $\boldsymbol{Y}_{t-1}$. Furthermore, it can be shown from the state and observation equations in (10) that

$$
p\left(y_{t} \mid \boldsymbol{Y}_{t-1}, \boldsymbol{S}_{t-1}^{(j)}, s_{t}=a_{i}\right) \sim N\left(\mu_{y_{t}}^{(j)}(i), \sigma_{y_{t}}^{2(j)}(i)\right)
$$

with mean and variance given by

$$
\begin{aligned}
\mu_{y_{t}}^{(j)}(i) & =E\left\{y_{t} \mid \boldsymbol{Y}_{t-1}, \boldsymbol{S}_{t-1}^{(j)}, s_{t}=a_{i}\right\} \\
& =a_{i}\left(P_{t} \boldsymbol{c}^{T} \boldsymbol{\mu}_{t-1}^{(j)}+Q_{t}\right) \\
\sigma_{y_{t}}^{2(j)}(i) & =\operatorname{Var}\left\{y_{t} \mid \boldsymbol{Y}_{t-1}, \boldsymbol{S}_{t-1}^{(j)}, s_{t}=a_{i}\right\} \\
& =\boldsymbol{c}^{T}\left(\boldsymbol{A} \boldsymbol{\Sigma}_{t-1}^{(j)} \boldsymbol{A}^{T}+\sigma_{u}^{2} \boldsymbol{b} \boldsymbol{b}^{H}\right) \boldsymbol{c}+\sigma_{n}^{2}
\end{aligned}
$$

where the quantities $\mu_{\boldsymbol{x}_{t}^{(j)}}$ and $\sigma_{\boldsymbol{x}_{t}^{2(j)}}$ in (27) can be computed recursively for the Extended Kalman equations given in (14). The trial distribution in (23) can be computed as follows:

$$
\begin{aligned}
p\left(s_{t}=a_{i} \mid \boldsymbol{Y}_{t}, \boldsymbol{S}_{t-1}^{(j)}\right)= & p\left(y_{t} \mid \boldsymbol{Y}_{t-1}, \boldsymbol{S}_{t-1}^{(j)}, s_{t}=a_{i}\right) \\
& \times P\left(s_{t}=a_{i} \mid \boldsymbol{Y}_{t-1}, \boldsymbol{S}_{t-1}^{(j)}\right) \\
\triangleq & \xi_{t, i}^{(j)}
\end{aligned}
$$

where it follows from (25) and (26) that

$$
\xi_{t, i}^{(j)}=\frac{1}{\pi \sigma_{y_{t}}^{2(j)}(i)} \exp \left(-\frac{\left\|y_{t}-\mu_{y_{t}}^{(j)}(i)\right\|^{2}}{\sigma_{y_{t}}^{2(j)}(i)}\right) P\left(s_{t}=a_{i}\right) .
$$

We now summarize the SMC blind data detection and phase noise estimation algorithm as follows:

Step 1- Initialization:

- Initialize the extended Kalman filter: Choose the initial mean and the covariance of the estimated $\boldsymbol{x}_{t}$ as

$$
\boldsymbol{\mu}_{-1}^{(j)}=\widehat{\boldsymbol{x}}_{-1 \mid-1}^{(j)}=0, \quad \boldsymbol{\Sigma}_{-1}^{(j)}=2 \boldsymbol{\Sigma} \quad j=1,2, \cdots, m
$$

where $\boldsymbol{\Sigma}$ is the stationary covariance of $\boldsymbol{x}_{t}$ and can be computed analytically from (7)

- Initialize the importance weights: All importance weights are initialized as $w_{-1}^{(j)}=1, j=1,2, \cdots, m$. Since the data symbols are are assumed to be independent, initial symbols are not needed be generated.

Step 2- Compute $\xi_{t, i}^{(j)}$ : For each $a_{i} \in A$ compute the $\mu_{y_{t}}^{(j)}(i)$, $\sigma_{y_{t}}^{2(j)}(i)$ and $\xi_{t, i}^{(j)}$ from (21), (22),(6),(7) and (24), respectively.

Step 3- Draw samples $s_{t}^{j}, j=1,2, \cdots, m$ Draw $s_{t}^{(j)}$ from the set $A$ with probabilities $P\left(s_{t}^{(j)}=a_{i}\right) \propto \xi_{t, i}^{(j)}, a_{i} \in A$. Append $s_{t}^{(j)}$ to $\boldsymbol{S}_{t-1}^{(j)}$ to obtain $\boldsymbol{S}_{t}^{(j)}$.

Step 4- Compute the importance weights:

$$
w_{t}^{(j)}=w_{t-1}^{(j)} \sum_{a_{i} \in A} \xi_{t, i}^{(j)} .
$$

Step 5-Detect the symbol $s_{t}$ : Detect the symbol $s_{t}$ from (17), (18) and (22).

Step 6-Update the a posteriori mean and variance of the phase noise: If the samples drawn up to time $t$ is $\boldsymbol{S}_{t}$ in Step 3, set

$$
\begin{aligned}
& \boldsymbol{\mu}_{t}\left(\boldsymbol{S}_{t}^{(j)}\right) \triangleq \boldsymbol{\mu}_{t}^{(j)}=\widehat{\boldsymbol{x}}_{t \mid t}^{(j)} \\
& \boldsymbol{\Sigma}_{t}\left(\boldsymbol{S}_{t}^{(j)}\right) \triangleq \boldsymbol{\Sigma}_{t}^{(j)}=\boldsymbol{M}_{t \mid t}^{(j)} \quad j=1,2, \cdots, m .
\end{aligned}
$$

and update according to the Kalman equations (14).

Step 7-Do the resampling as described in Section IV.

\section{Resampling Method}

A major problem in the practical implementation of the SMC method described so far is that after a few iteration most of the importance weights have negligible values that is $w_{t}^{(j)} \approx 0$. A relatively small weight implies that the sample is drawn far from the main body of the posterior distribution and has a small contribution in the final estimation. Such sample is said to be ineffective. The SMC algorithm becomes ineffective if there are too many ineffective samples. The common solution to this problem is resampling. Resampling is a an algorithmic step that stochastically eliminates those samples with small weights. Basically, the resampling method takes the samples, to be generated sequentially $\Xi_{t}=\left\{\boldsymbol{S}_{t}^{(j)}, \boldsymbol{\mu}_{t}^{(j)}, \boldsymbol{\Sigma}_{t}\right\}_{j=1}^{m}$ with corresponding weights $\left\{w_{t}^{(j)}\right\}_{j=1}^{m}$ as an input and generates a new set of samples $\widetilde{\Xi_{t}}=\left\{\widetilde{\boldsymbol{S}}_{t}^{(j)}, \widetilde{\boldsymbol{\mu}}_{t}^{(j)}, \widetilde{\boldsymbol{\Sigma}}_{t}\right\}_{j=1}^{m}$ with equal weights, i.e $\left\{w_{t}^{(j)}=1 / m\right\}_{j=1}^{m}$, assuming they are normalized to $\sum_{j=1}^{m} w_{t}^{(j)}=1$. A simple procedure to achieve this 
goal is, for each $j=1,2, \cdots, m$, to choose $\left(\widetilde{\boldsymbol{S}}_{t}^{(j)}, \widetilde{\boldsymbol{\mu}}_{t}^{(j)}, \widetilde{\boldsymbol{\Sigma}}_{t}\right)=$ $\left(\boldsymbol{S}_{t}^{(j)}, \boldsymbol{\mu}_{t}^{(i)}, \boldsymbol{\Sigma}_{t}\right)$ with probability $w_{t}^{(i)}$.

In this paper, a resampling technique suggested by [11] is employed. This technique forms a new set of weighted samples $\widetilde{\Xi_{t}}=\left\{\widetilde{\boldsymbol{S}}_{t}^{(j)}, \widetilde{\boldsymbol{\mu}}_{t}^{(j)}, \widetilde{\boldsymbol{\Sigma}}_{t}\right\}_{j=1}^{m}$ according to the following algorithm. (assume that $\sum_{j=1}^{m} w_{t}^{j}=m$ )

1) For $j=1,2, \cdots, m$, retain $\ell_{j}=w_{t}^{j}$ copies of the samples $\left(\boldsymbol{S}_{t}^{(j)}, \mu_{t}^{(i)}, \sigma_{t}\right)$. Denote $L_{r}=m-\sum_{j=1}^{m} \ell_{j}$.

2) Obtain $L_{r}$ i.i.d. draws from the original sample set $\left\{\left(\boldsymbol{S}_{t}^{(j)}, \mu_{\boldsymbol{x}_{t}}^{(i)}, \sigma_{\boldsymbol{x}_{t}}\right)\right\}_{j=1}^{m}$, with probabilities proportional to $\left(w_{t}^{j}-\ell_{j}\right), j=1,2, \cdots, m$.

3) Assign equal weights, that is, set $w_{t}^{j}=1$, for each new sample.

It is shown in [11] that the samples drawn by the above procedure are properly weighted with respect to $p\left(\boldsymbol{S}_{t} \mid \boldsymbol{Y}_{t}\right)$, provided that $m$ is sufficiently large. Note that resampling at every time step is not needed in general. In one way the resampling can be done every $k_{0}$ recursions where $k_{0}$ is a prefixed resampling interval. On the other hand, the resampling can be carried out whenever the effective sample size, approximated as

$$
\widehat{N}_{e f f}=\frac{1}{\sum_{j=1}^{m}\left(w_{t}^{j}\right)^{2}} \leq m
$$

goes below a certain threshold, typically a fraction of $\mathrm{m}$.

\section{Simulation Results}

In this section, we provide some computer simulation examples to demonstrate the performance of the proposed SMC receivers. The residual phase noise is modelled by the output of a first-order digital PLL. Specifically as pointed out in Section2 , the coefficients $\left\{\theta_{t}\right\}$ is modelled by the following ARMA process

$$
\theta_{t}=(1-a) \theta_{t-1}+u_{t}
$$

where $0<a<1$ is a system parameter describing the loop filter transfer function. $a$ is chosen such that $\operatorname{Var}\left\{\theta_{t}\right\}=1$. The driver noise $u_{t}$ is assumed to be zero-mean and the variance $\sigma_{u}^{2}=0.1$. It is assumed that BPSK modulation is employed. In order to demonstrate the performance of the adaptive SMC approach, we first present the performance (in terms of bit-errorrate (BER)) of the proposed SMC approach together with theoretical lower bound. The uncoded BER performance of this adaptive approach is plotted in Fig. 1. The performance of the Kalman filter part to track the phase process based on the symbols provided by SMC is also shown in Fig. 2 for a $10 \mathrm{~dB}$ and $20 \mathrm{~dB}$ SNRs.

\section{Conclusions}

We have developed a new adaptive Bayesian approach for blind phase noise estimation and data detection based on SMC. The optimal solutions to joint symbol detection and phase noise estimation problem is computationally prohibitive to implement by conventional methods. Thus the proposed sequential approach offers an novel and powerful approach to tackling this

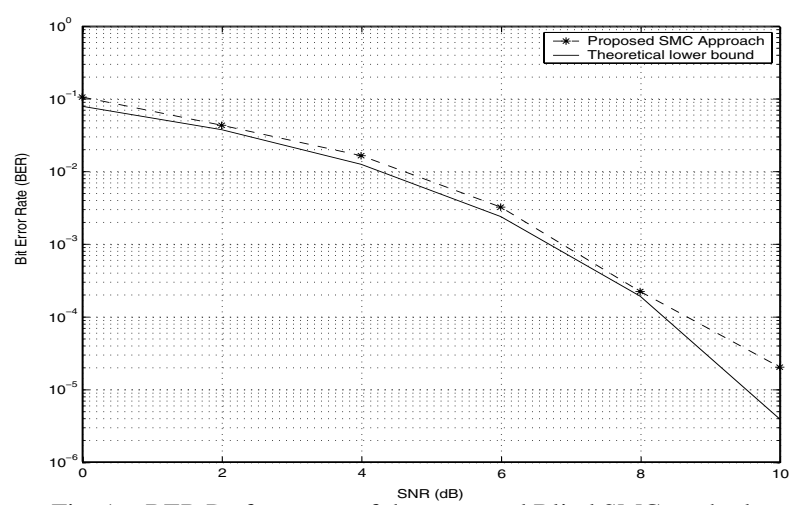

Fig. 1. BER Performance of the proposed Blind SMC method
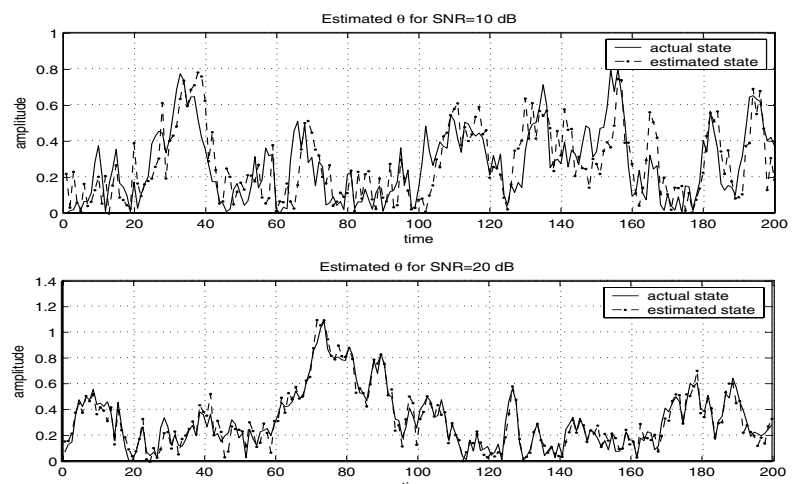

Fig. 2. Actual and Estimated ${ }^{\text {timh }}$ phase for two different SNRs

problem at a reasonable computational cost. It is shown through simulations that the performance of the proposed blind SMC algorithm can track the phase remarkably close to the true phase values.

\section{REFERENCES}

[1] H. Meyr, M. Moeneclaey and S.A. Fechtel, Digital Communications Receivers, John Wiley, New York, 1998.

[2] C. N. Georghiades and J.C. Han, "Optimum decoding of trellis coded modulation in the presence of phase noise", Proc. 1990 Int. Symp. on Information Theory and Its Applications (ISITA'90) Hawaii, Nov. 1990.

[3] A. T. Huq, E. Panayirci and C. N. Georghiades, " ML NDA carrier phase recovery for OFDM systems with M-PSK signaling", Proc. IEEE Int. Conf. on Communications (ICC'99), June 6-10, 1999, Vancouver, Canada.

[4] E. Panayırcı and C. N. Georghiades," Non-data-aided ML carrier frequency and phase synchronization in OFDM systems", European Transactions on Telecommunications (ETT), 2000.

[5] A. P. Dempster, N. M. Laird and D. B. Rubin,"Maximum likelihood from incomplete data via the EM algorithm",Ann. Roy. Stat. Soc., pp. 1-38, December 1977.

[6] M. Pitt and N. Shephard," Filtering via simulation: auxiliry particle filter",J. Amer. Statist. Soc. B , no.63, 2001.

[7] E. Panayırc1, H. A. Cirpan and M.Moeneclaey, "A Sequential Monte Carlo Method for Blind Phase Noise Estimation and Data Detection," 13th European Signal Processing Conference EUSIPCO2005.

[8] S. Geman and D. Geman," Stochastic relaxation, Gibbs distribution and the Bayesian restoration of images",IEEE Trans. on Pattern Analysis nad Machine Intelligence vol. 6 ,pp. 721-741, 1984.

[9] S.M. Kay, "Fundamentals of Statistical Signal Processing: Estimation Theory," Prentice Hall 1993.

[10] Z. Yang and X. Wang, " A sequential Monte Carlo blind receiver for OFDM systems in frequency-selective fading channels",IEEE Trans. Signal Proc.,vol. 50, no. 2, pp.271-280, Feb. 2003.

[11] R. Chen, X. Wang and J. S. Liu, " Adaptive joint detection and decoding in flat-fading channels via mixture Kalman filering", IEEE Trans.Information Theo.,vol. 46, no. 6, pp.2070-2094, Sept. 2000.

[12] J. S. Liu and R. Chen,"Blind deconvolution via sequential imputations", J. Amer. Statist. Assoc., vol. 90, pp. 567-576, 1995. 